\title{
In Situ ESEM Investigation of the drying of demineralized dentin
}

\author{
V.M.Dusevich, A.G.Glaros, J.D.Eick \\ University of Missouri - Kansas City, School of Dentistry, Kansas City, MO 64155
}

For proper adhesion of dental composites dentin should be etched with acids prior to the application of dental primers and/or adhesives. The etched and rinsed layer of dentin consists mostly of a collagen network in water. Modern dental adhesives exhibit better bonding to wet dentin [1]. Therefore, if the demineralized layer is dried before adhesive application, the clinical performance of the composite may be compromised.

Five specimens of human dentin were etched for 15 seconds with $37 \%$ phosphoric acid and another five specimens with 10:3 etchant (10 parts citric acid to 3 parts ferric chloride in water). After etching, each specimen was washed with water for 30 seconds and immediately split (to have the profile of the etched layer for observation), placed in a field emission ESEM (XL30, FEI Company, OR USA) and observed at 5.6 Torr pressure and $5^{\circ} \mathrm{C}$. For each specimen, the 3 different fields of view were photographed immediately after pumping was finished, and 10, 20,30 and 40 minutes later. Then the specimen was completely dried (at $8^{\circ} \mathrm{C}$ and 0.1 Torr for 10 minutes) and final set of micrographs was taken. Micrographs of dehydrating dentin were superimposed with the original micrograph of the same area (Fig. 1a), so that the decrease of the etched dentin area (black shade on fig. 1b) and the original area (black plus gray shade on fig. 1b) could be determined and measured. Statistical tests were performed at $p \leq 0.05$. Results of measurements are presented in a Table 1. An analysis of variance with repeated measures showed significant effects for Time, Etchant, and the interaction of Time and Etchant. The height of the demineralized zone decreased as drying time progressed. Demineralized zones were consistently larger for the 10:3 etchant, and also showed greater rates of collapse than the phosphoric acid treated specimens. Thickness change was greatest in the first 10 minutes in the ESEM chamber. Drying specimens after 40 min exposure to the ESEM chamber lead to additional shrinkage, indicating that even after 40 min specimens were not completely dehydrated. A smaller shrinkage of the dried demineralized layer created with the 10:3 etchant could be the result of stabilization by ferric chloride of the collagen network.

Profiles of the etched layer of the specimens, dried in the ESEM chamber (Fig. 2a), did not show the collagen network as did the specimens dehydrated in gradated alcohol solutions (Fig. 2b). The micrograph of the air-dried specimen (Fig. 3a) showed an amorphous layer on top of collagen fibers in contrast to the previously observed surface layer (Fig. 3b), consisting of collagen fibers[2]. The amorphous layer could consist of dried proteins from the dentin, which could completely hide underlying collagen fibers and form a featureless surface (Fig.4a) in contrast to specimens dehydrated in alcohol, where the proteins are washed out (Fig.4b). An earlier assumption that this layer is created by destruction caused by surface tension [3] can not explain the reversible character of the layer formation, i.e. dehydration in alcohol of the specimen dried in the ESEM for 40 minutes (Fig. 4c) dissolved the protein layer and presented the same surface structure as initially dehydrated specimens [4].

[1] J.D.Eick et al., Crit Rev Oral Biol Med (1997) 8(3) (1997) 306-335

[2] D.H.Pashley et al., Quintessence Int, 24(9) (1993) 618-631

[3] A.J.Gwinnet, Dent Mater, 10 (1994) 150-155

[4] This project was supported in part by NIDCR research grant DE09696 


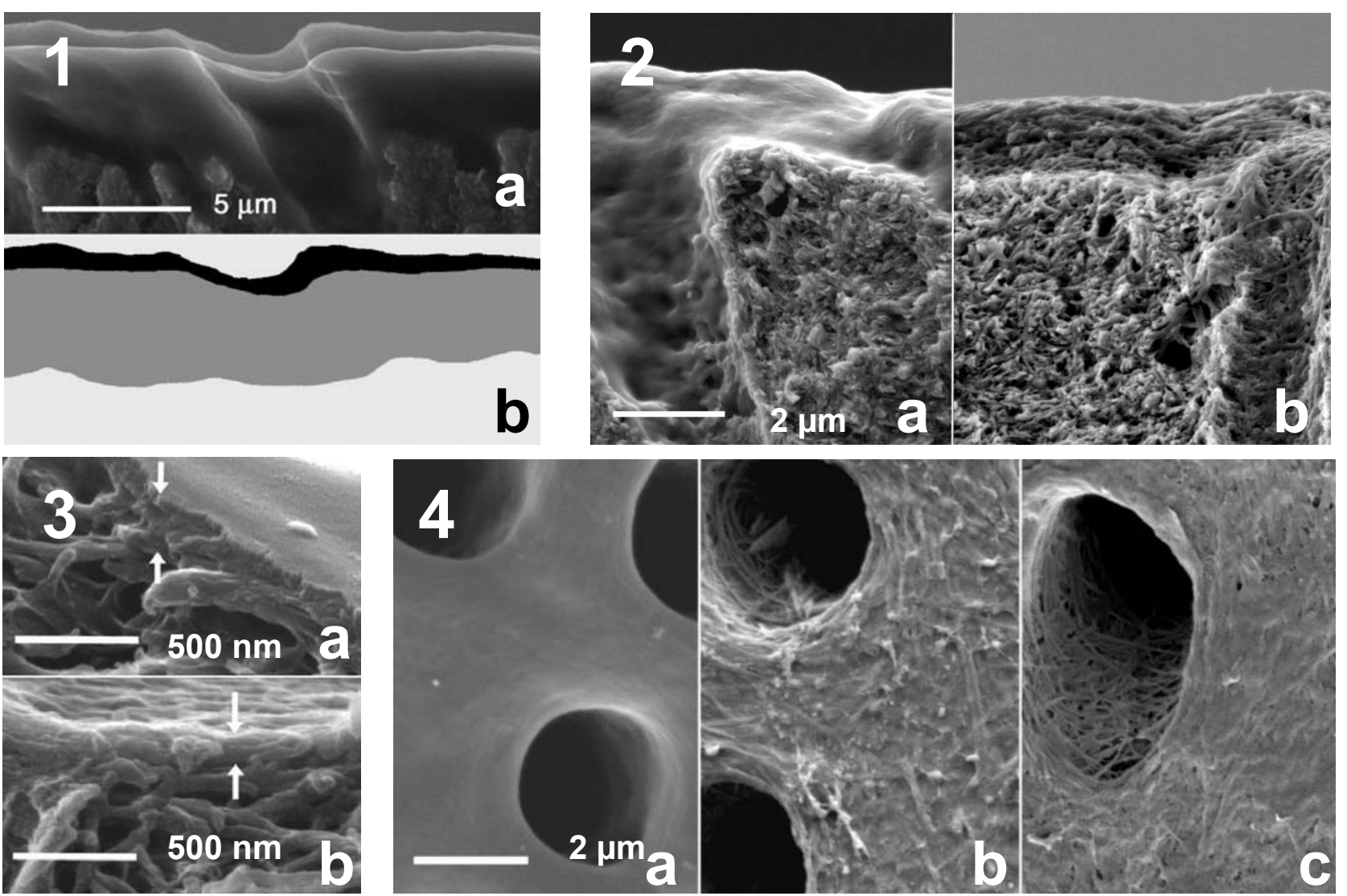

Figure 1. Original micrograph of etched dentin in the environmental mode, superimposed with the micrograph of the same place after specimen dried for 40 minutes (a), and the image prepared for measurements (b).

Figure 2. Profile of the demineralized layer of the specimen, dried in the ESEM chamber (a), and the profile of the alcohol-dehydrated specimen (b).

Figure 3. Surface layer of the specimen, dried in the ESEM chamber (a), and of the alcohol dehydrated specimen (b).

Figure 4. Demineralized layer (view from the top) of the specimen dried in the ESEM chamber (a), alcohol dehydrated specimen (b), and specimen dried in the ESEM chamber for 40 minutes and then alcohol dehydrated (c).

Table 1. Decrease in the thickness of demineralized dentin layer due to dehydration in the ESEM specimen chamber

\begin{tabular}{|c|c|c|c|c|}
\hline \multirow{2}{*}{$\begin{array}{c}\text { Time in the } \\
\text { chamber, min }\end{array}$} & \multicolumn{2}{|l|}{ Relative thickness decrease, $\%$} & \multicolumn{2}{l|}{ Thickness of etched layer, $\mu \mathrm{m}$} \\
\cline { 2 - 5 } & $10: 3$ etchant & Phosphoric acid & $10: 3$ etchant & Phosphoric acid \\
\hline 0 & 0 & 0 & $3.21 \pm 0.42$ & $1.10 \pm 0.13$ \\
10 & $12.4 \pm 5.2$ & $23.3 \pm 10.1$ & $2.82 \pm 0.39$ & $0.78 \pm 0.16$ \\
20 & $17.7 \pm 6.2$ & $26.0 \pm 8.8$ & $2.65 \pm 0.41$ & $0.75 \pm 0.15$ \\
30 & $19.4 \pm 6.0$ & $28.9 \pm 8.8$ & $2.60 \pm 0.40$ & $0.72 \pm 0.16$ \\
40 & $20.2 \pm 5.8$ & $31.3 \pm 7.8$ & $2.57 \pm 0.39$ & $0.70 \pm 0.14$ \\
\hline Dried & $26.1 \pm 5.9$ & $35.6 \pm 7.7$ & $2.38 \pm 0.36$ & $0.65 \pm 0.14$ \\
\hline
\end{tabular}

